\title{
High-speed Scanner with Nanometer Resolution Using a Hybrid Reluctance Force Actuator
}

\author{
Shingo Ito ${ }^{* a)} \quad$ Non-member, Stefan Troppmair ${ }^{* *}$ Non-member \\ Francesco Cigarini* Non-member, Georg Schitter* ${ }^{*} \quad$ Non-member
}

(Manuscript received April 16, 2018, revised Aug. 20, 2018)

\begin{abstract}
This paper proposes a high-precision, high-speed scanner using a hybrid reluctance actuator, which can be stronger than conventionally used comparable Lorentz force actuators. For compactness, its mover is guided by flexures and laterally moved by a hybrid reluctance actuator with a voltage amplifier. To reject disturbances such as thermal drift and hysteresis, the scanner is regulated by cascade control, for which parasitic resonances are damped partially mechanically. As a result, the closed-loop system realizes a high control bandwidth of $3.5 \mathrm{kHz}$ and a high positioning resolution of $0.8 \mathrm{~nm}$ at a static point. For high-speed scanning motion, modeling-free inversion-based iterative control (IIC) is proposed to be combined with the cascade control as the scanner's feedforward controller. Experiments demonstrate that the scanner with the cascade control realizes a $2 \mu \mathrm{m}$ triangular motion at $400 \mathrm{~Hz}$ with a tracking error of $101 \mathrm{~nm}$, and the modeling-free IIC successfully decreases this relatively large error by a factor of 26 to $3.8 \mathrm{~nm}$. Consequently, this paper clearly demonstrates that the proposed scanner with the hybrid reluctance actuator can realize high-precision, high-speed scanning motion.
\end{abstract}

Keywords: high-precision actuators, hybrid reluctance actuator, nanopositioning, motion control

\section{Introduction}

Many applications require high-precision motion. Some examples are 3D printers ${ }^{(1)}$, atomic force microscopes (AFMs) ${ }^{(2)}$, vibration isolators ${ }^{(3)}$ and data storage devices (e.g. Blu-ray players and hard disk drives) ${ }^{(4)}$. When compact actuators are required with nanometer resolution for these systems, piezoelectric actuators or Lorentz actuators (e.g. voice coil actuators) are usually guided by flexures.

In contrast to piezoelectric actuators, flexure-guided Lorentz actuators realize a control bandwidth that is significantly higher than the first dominant resonant frequency by design. Such actuators are defined as "low-stiffness actuators," and they exhibit good vibration isolation performance ${ }^{(5)}$. Consequently, they are capable of positioning with nanometer resolution even without external vibration isolation $^{(6)}$ and are applied to optical disk drives ${ }^{(7)}$ and AFM ${ }^{(8)}$. Low-stiffness actuators are also beneficial to achieve a large actuation range ${ }^{(9)}$. However, Lorentz actuators have a disadvantage that their motor constant (i.e. force-to-current ratio) is relatively small ${ }^{(10)}$. This is problematic to generate highspeed scanning motion, for example for AFMs, since it requires a high acceleration force. A large current for a required force increases the temperature of the Lorentz coils, resulting

\footnotetext{
a) Correspondence to: Shingo Ito. E-mail: ito@acin.tuwien.ac.at

* Christian Doppler Laboratory for Precision Engineering for Automated In-Line Metrology, Automation and Control Institute (ACIN), TU Wien

Gusshausstrasse 27-29, A-1040 Vienna, Austria

** Automation and Control Institute (ACIN), TU Wien

Gusshausstrasse 27-29, A-1040 Vienna, Austria
}

in thermal drift or expansion to impair the precision of the system. As a countermeasure, a cooling system ${ }^{(1)}$ or thermal isolation ${ }^{(12)}$ is incorporated. However, a low-stiffness actuator with a high motor constant is highly desired to decrease the Joule heat, in order to develop a compact high-speed scanner without wasting energy.

As an alternative replacing Lorentz actuators, reluctance actuators produce a large force that is proportional to the square of the coil current ${ }^{(10)}$. Although such a nonlinearity can be compensated by measuring the magnetic flux ${ }^{(13)}$, the measurement noise may degrade the positioning resolution. In contrast, hybrid reluctance actuators generate a force proportional to the current by using a permanent magnet, achieving a motor constant higher than Lorentz actuators ${ }^{(10)}$. For the advantages, hybrid reluctance actuators have been applied to fast tool servos ${ }^{(14)(15)}$, active vibration isolators ${ }^{(16)}$ and steering mirrors ${ }^{(17)(18)}$. However, hybrid reluctance actuators exhibit mechanical modes and hysteresis ${ }^{(10)}$ together with eddy current influencing the dynamics ${ }^{(18)}$. They are a concern to achieve desired performance, dependent on applications.

In order to utilize its high motor constant, this paper proposes a short-stroke high-speed scanner with nanometer resolution based on a hybrid reluctance actuator. To demonstrate the suitability of the hybrid reluctance actuator for high-speed scanning with nanometer resolution, the dynamics and disturbances are compensated by cascade feedback control regulating the coil current and the mover position. Additionally, to accurately track a high-speed scanning trajectory, a modeling-free learning algorithm is applied as feedforward control to the the hybrid reluctance scanner.

The rest of the paper is organized as follows. Section 2 


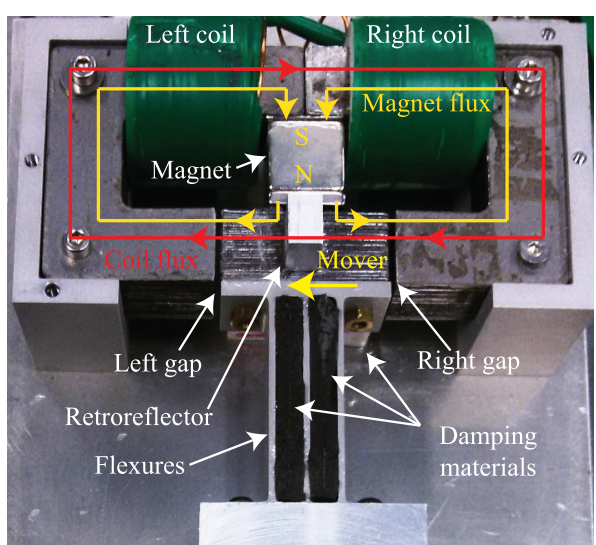

Fig. 1. Photograph of the high-speed scanner with a hybrid reluctance actuator, where the red and yellow paths denote the magnetic flux due to the coils and the permanent magnet, respectively

describes the high-speed scanner using a hybrid reluctance actuator, which is modeled in Section 3. After current control is discussed in Section 4, the scanner is experimentally analyzed in Section 5, for position control design in Section 6. Section 7 introduces a high-speed trajectory, for which feedforward control is designed in Section 8. Section 9 presents experimental results. Section 10 concludes the paper.

\section{System Description}

Figure 1 shows a high-speed scanner with a hybrid reluctance actuator. Due to their linearity without backlash and friction, leaf-spring flexures are selected to laterally guide the mover. Around the mover, a C-core is fixed to the mechanical ground as the stator together with a permanent magnet and two identical coils. Both the mover and the stator are made from laminated electrical steel sheets (EN10025S235JR), which reduce the eddy current in comparison to solid blocks ${ }^{(18)}$. The achievable closed-loop bandwidth is typically restricted by the second and higher mechanical resonances in the case of low-stiffness actuators. To achieve a high bandwidth, these modes are suppressed by inserting a rubber material (B30, Rotring, Hamburg, Germany) beneath the mover and by adding Sorbothane sheets (SB12, Thorlab, New Jersey, USA) between the flexures.

The lateral motion of the mover is measured by an interferometer (10899A, Agilent Technologies, Santa Clara, USA) with a resolution of $1.25 \mathrm{~nm} / \mathrm{bit}$. As its target, a retroreflector (43-305, Edmund optics, Barrington, USA) is used and fixed to the mover. The coils are electrically connected in series and driven by a custom-made voltage amplifier with a current monitor. The amplifier can output a current up to $\pm 2 \mathrm{~A}$, and the output voltage is less than $\pm 30 \mathrm{~V}$. To implement control algorithms, a prototyping control system with an FPGA (DS5203, dSpace GmbH, Paderborn, Germany) and CPU (DS1005) is used. The FPGA communicates with the amplifier via a 14-bit DAC/ADC and with the interferometer via a 36-bit parallel bus, as shown in Fig. 2. The sampling frequency of the FPGA is fixed to $10 \mathrm{MHz}$ to implement the current loop in Section 4. Although the sampling frequency of the CPU is set to about $60 \mathrm{kHz}$ to implement position control only in Section 6, it has to be decreased to

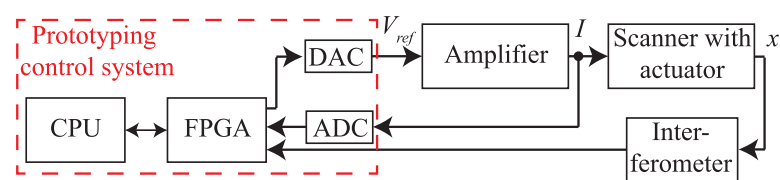

Fig. 2. System architecture, where $V_{\text {ref }}, I, x$ are the reference input to the amplifier, the coil current and the nanopositioner position, respectively. The current is measured by the current monitor of the amplifier

$20 \mathrm{kHz}$ to implement both feedback and feedforward control for Section 8 and 9.

\section{System Modeling}

As indicated by the yellow lines in Fig. 1, the permanent magnet creates magnetic flux through the left and right gaps around the mover. Similarly, the coils generate flux as shown by the red lines in Fig. 1. The coil flux does not penetrate the permanent magnet because its reluctance is as large as the air. The superimposed flux of the left gap is stronger than the right. Due to to the unbalanced flux, a lateral actuation force is generated for scanning motion. Notice that the hybrid reluctance actuator is bidirectional, unlike typical reluctance actuators and some piezoelectric actuators.

Hybrid reluctance actuators' force $F$ can be described by a motor constant $k_{m}$ and actuator magnetic stiffness $k_{a}^{(15)(17)}$

$$
F(t)=k_{m} I(t)+k_{a} x(t),
$$

where $I$ and $x$ are the coil current and the mover position, respectively, and $k_{m}$ and $k_{a}$ of the actuator in Fig. 1 are about $8.5 \mathrm{~N} / \mathrm{A}$ and $30 \mathrm{kN} / \mathrm{m}$, respectively ${ }^{(19)}$. Since the mover is guided by the flexures, the scanner is modeled as a damped mass-spring system ${ }^{(10)}$

$$
F(t)=m \ddot{x}(t)+c \dot{x}(t)+k x(t), \cdots
$$

where the mover mass $m$ is $55 \mathrm{~g}$. The damping coefficient $c$ and stiffness $k$ are due to the damping materials and the flexures $^{(20)}$. The Laplace transform of (1) and (2) gives the transfer function $P_{a}(s)$ from $I$ to $x$

$$
P_{a}(s)=\frac{x(s)}{I(s)}=\frac{g_{p}}{\frac{s^{2}}{\omega_{n}^{2}}+2 \zeta \frac{s}{\omega_{n}}+1}
$$

where the plant gain $g_{p}$, the natural frequency $\omega_{n}$ and the damping ratio $\zeta$ are

$$
g_{p}=\frac{k_{m}}{k-k_{a}}, \omega_{n}=\sqrt{\frac{k-k_{a}}{m}}, \zeta=\frac{c}{2 \sqrt{m\left(k-k_{a}\right)}} .
$$

Note that the scanner is designed to be $k>k_{a}$ with the flexures having a stiffness of $80 \mathrm{kN} / \mathrm{m}$ for stability within the target high-speed scanning range.

\section{Current Control}

Equation (1) shows that the force is related to the current. Therefore, the coil current is regulated by feedback control before position control design, for high closed-loop bandwidth, as commonly seen in servo systems ${ }^{(21)}$. The current $I$ can be related to the amplifier's reference input $V_{r e f}$ 

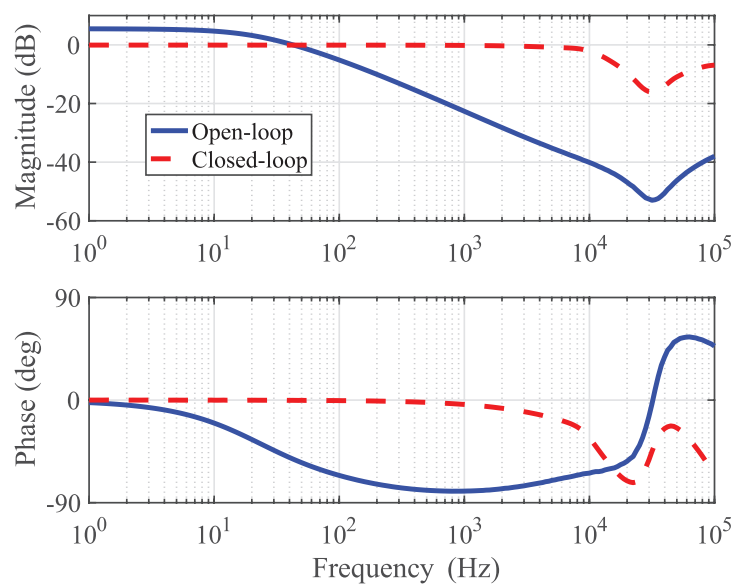

Fig. 3. Measured Bode plots of the current $I$. The blue solid line shows a response from the amplifier's reference $V_{\text {ref }}$ in open loop while the red dashed line does from the current reference $I_{r e f}$ in the closed loop (i.e. the complementary sensitivity function)

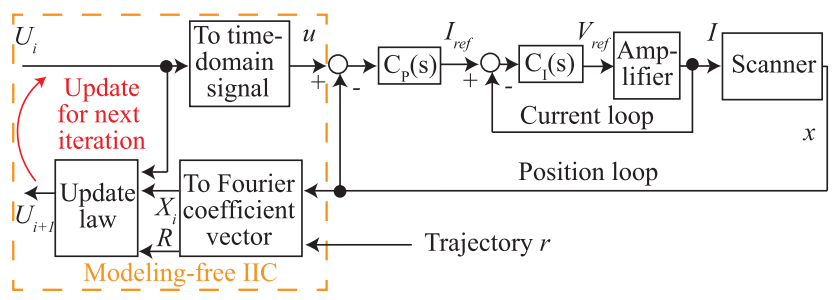

Fig. 4. Block diagram of the scanner and its control

$$
P_{e}(s)=\frac{I(s)}{V_{r e f}(s)}=\frac{g_{a m p}}{L s+R},
$$

where the amplifier gain $g_{a m p}$ is three. The resistance $R=1.64 \Omega$ and the inductance $L=0.01 \mathrm{H}$ of the connected coils are measured by an RLC meter. For control design, the current-voltage relation formulated by (5) is investigated by measuring a frequency response from $V_{\text {ref }}$ to $I$, as shown by the blue solid line in Fig. 3. Due to the pole resulting from the coils' impedance in (5), the response shows low-pass characteristics with a $-3 \mathrm{~dB}$ bandwidth of about $25 \mathrm{~Hz}$. At high frequencies beyond $30 \mathrm{kHz}$, the magnitude increases, which may be due to the parasitic dynamics of the amplifier and the coils.

As the feedback controller $C_{I}(s)$ shown in Fig. 4, a PI controller with a low-pass filter is used to compensate for the pole and the parasitic dynamics. The controller is implemented by the FPGA, and their gains are tuned to achieve a highest possible closed-loop bandwidth without unstable oscillation of the output current. The resulting frequency response from the current reference $I_{\text {ref }}$ to $I$ is measured, as shown by the red dashed line in Fig. 3, which indicates that $C_{I}(s)$ increases the $-3 \mathrm{~dB}$ bandwidth by a factor of 440 to $11 \mathrm{kHz}$.

\section{System Analysis}

5.1 System Identification For position control design, a frequency response is measured from the current reference $I_{r e f}$ to the position $x$ with and without the damping materials (cf. Fig. 1), as shown in Fig. 5. The first mechanical resonance with the damping materials at $210 \mathrm{~Hz}$ is the mode predicted by the model $P_{a}(s)$. Its frequency and peak
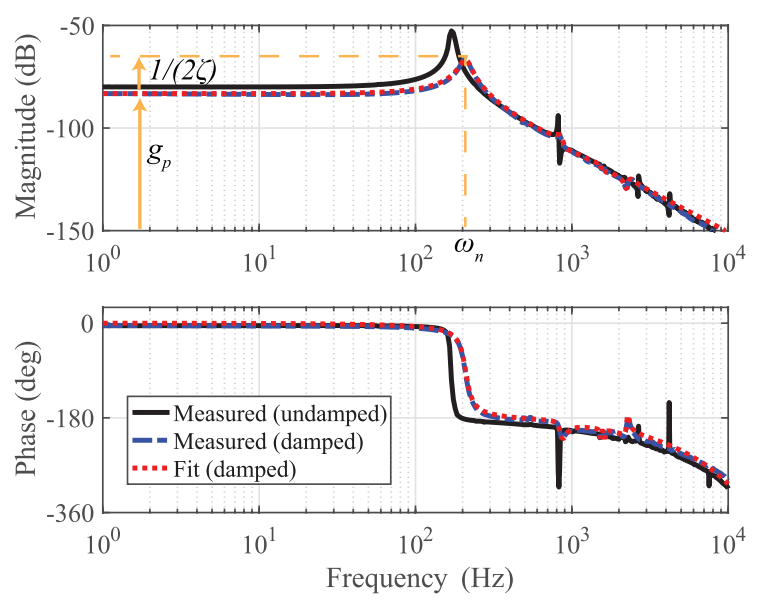

Fig. 5. Measured Bode plot from the current reference $I_{r e f}$ to the position $x$ with and without damping materials. The extended model (6) is fit to the response with the damping materials

gain can be approximated by $\omega_{n}$ and $g_{p} /(2 \zeta)$, respectively, as indicated in Fig. 5.

The measured response also shows dynamics that are not modeled by the analytical model $P_{a}(s)$. Figure 5 shows that high-frequency mechanical modes (e.g. at $820 \mathrm{~Hz}$ ) are suppressed to some extent by the damping materials. However, they are still visible even with the damping materials. Additionally, the phase plot shows a large phase lag beyond $800 \mathrm{~Hz}$, which might be partially due to the residual eddy current $^{(18)}$. Because these dynamics influence position control design, they are modeled by extending the analytical model as follows

$$
P(s)=P_{a}(s) P_{p}(s) e^{-s T_{d}},
$$

where the delay $T_{d}$ models the phase lag, and the highfrequency mechanical modes are captured by ${ }^{(22)}$

$$
P_{p}(s)=g_{p p} \prod_{q=1}^{N} \frac{s^{2}+2 \zeta_{n q} \omega_{n q} s+\omega_{n q}^{2}}{s^{2}+2 \zeta_{d q} \omega_{d q} s+\omega_{d q}^{2}}, \cdots \ldots \ldots \ldots
$$

where $g_{p p}$ and $N$ are the gain and the number of the modes, respectively. The parameters of $P(s)$ are determined by fitting it to the measured Bode plot, as shown in Fig. 5. During the fitting, $N$ is set to 4 to capture the mechanical modes up to about $2.3 \mathrm{kHz}$. Beyond that frequency, the phase lag dominates the frequency response, restricting the achievable control bandwidth (cf. Section 6). Figure 5 shows that $P(s)$ captures the measured frequency response very well. Table 1 lists the identified parameter values.

5.2 Nonlinearity In addition to the dynamics evaluated in the previous section, nonlinearity can impair the performance for high-precision motion. In the case of the hybrid reluctance actuator, the flux in the electrical steel varies, and the hysteresis between the flux and the coil current is a concern (cf. B-H hysteresis loop ${ }^{(23)}$ ). Therefore, it is evaluated by measuring the mover position $x$ when a $1 \mathrm{~Hz}$ sine wave is used as the current reference $I_{r e f}$.

The results are shown by the black solid line in Fig. 6, where the red dotted line is a linear function fit to the measured curve as the trend line for comparison. From the 
Table 1. Identified values of the extended model $P(s)$

\begin{tabular}{|cc|cc|}
\hline Parameter & Value & Parameter & Value \\
\hline$g_{p}$ & $-83.2 \mathrm{~dB}$ & $T_{d}$ & $35 \mu \mathrm{s}$ \\
$\omega_{n}$ & $2 \pi \times 210 \mathrm{rad} / \mathrm{s}$ & $\zeta$ & 0.075 \\
$g_{p p}$ & $0 \mathrm{~dB}$ & - & - \\
$\omega_{n 1}$ & $2 \pi \times 880 \mathrm{rad} / \mathrm{s}$ & $\zeta_{n 1}$ & 0.068 \\
$\omega_{n 2}$ & $2 \pi \times 1525 \mathrm{rad} / \mathrm{s}$ & $\zeta_{n 2}$ & 0.010 \\
$\omega_{n 3}$ & $2 \pi \times 1630 \mathrm{rad} / \mathrm{s}$ & $\zeta_{n 3}$ & 0.010 \\
$\omega_{n 4}$ & $2 \pi \times 2265 \mathrm{rad} / \mathrm{s}$ & $\zeta_{n 4}$ & 0.015 \\
$\omega_{d 1}$ & $2 \pi \times 854 \mathrm{rad} / \mathrm{s}$ & $\zeta_{d 1}$ & 0.052 \\
$\omega_{d 2}$ & $2 \pi \times 1520 \mathrm{rad} / \mathrm{s}$ & $\zeta_{d 2}$ & 0.010 \\
$\omega_{d 3}$ & $2 \pi \times 1625 \mathrm{rad} / \mathrm{s}$ & $\zeta_{d 3}$ & 0.010 \\
$\omega_{d 4}$ & $2 \pi \times 2275 \mathrm{rad} / \mathrm{s}$ & $\zeta_{d 4}$ & 0.020 \\
\hline
\end{tabular}

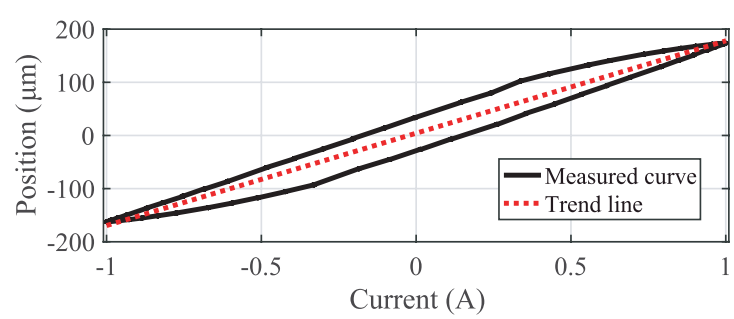

Fig. 6. Hysteresis measured with a $1 \mathrm{~Hz}$ sinusoidal coil current, to which a linear function is fit as the trend line

measured data, a hysteresis nonlinearity of $12 \%$ is calculated, which needs to be compensated for high-precision motion. Note that this nonlinearity is comparable to other highprecision actuators, for example, piezoelectric stack actuators that can have hysteresis up to about $20 \%{ }^{(24)}$.

\section{Position Control}

6.1 Design Rejecting disturbances such as thermal drift and floor vibrations, feedback control is desired for high positioning resolution. For this purpose, a position loop with a feedback controller $C_{P}(s)$ is designed as shown in Fig. 4. The controller $C_{P}(s)$ consists of the inversion of $P_{p}(s)$ to compensate for the high-frequency mechanical modes for a high closed-loop bandwidth. Additionally, $C_{P}(s)$ includes a tamed PID controller ${ }^{(10)} C_{P I D}(s)$ to compensate for a steadystate error due to the hysteresis and to provide a phase lead for high bandwidth, as follows

$$
C_{P}(s)=P_{p}^{-1}(s) C_{P I D}(s),
$$

The gains of $C_{P I D}(s)$ are tuned to ensure a phase margin of at least about 30 degrees for stability and to increase the openloop cross-over frequency as high as possible, resulting in

$$
\begin{aligned}
C_{P I D}(s)= & 3.697 \times 10^{5}+\frac{4.812 \times 10^{8}}{s} \\
& +\frac{98 s}{2.7 \times 10^{-5} s+1} . \cdots \cdots
\end{aligned}
$$

Figure 7 simulates the open-loop transfer function, using the designed $C_{P}(s)$ and the measured frequency response function in Fig. 5. The simulation shows the open-loop crossover frequency at $1.9 \mathrm{kHz}$ with a phase margin of 30 degrees. The frequency is significantly higher than the first resonant frequency of $210 \mathrm{~Hz}$. Such a system is categorized to lowstiffness actuators with high vibration isolation for highprecision positioning ${ }^{(5)}$. Note that the open-loop transfer function includes only one integrator, and the resulting position loop is Type $1^{(25)}$.

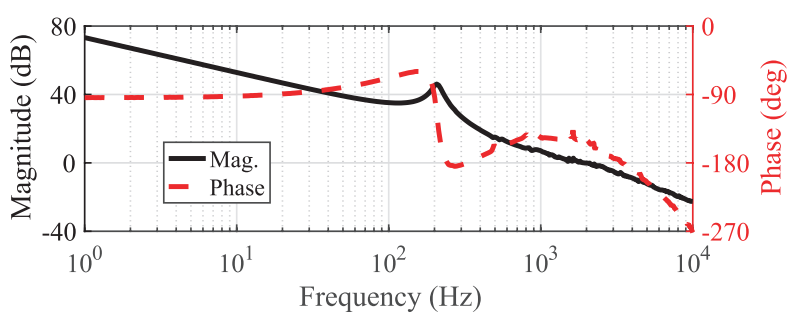

Fig. 7. Simulated open-loop transfer function with the designed $C_{P}(s)$ and the measured frequency response in Fig. 5

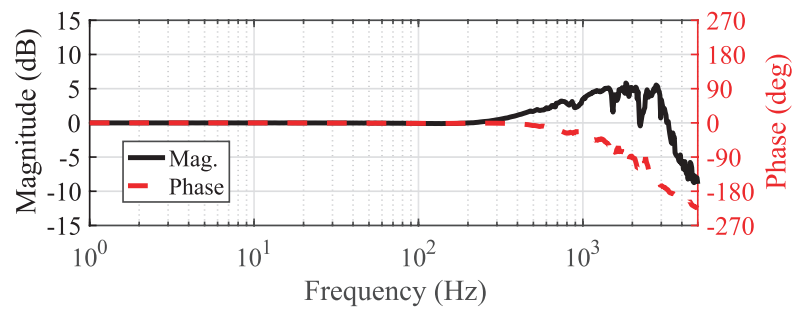

Fig. 8. Measured complementary sensitivity function of the position loop, which is the transfer function from $u$ to $x$ in Fig. 4
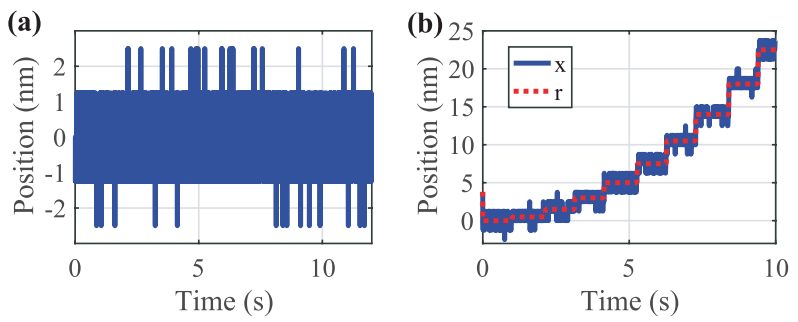

Fig. 9. Measured position $x$ when the cascade control is turned on (a) for the position reference $u=0$ and (b) for step inputs with different step height

6.2 Validation For validation, $C_{P}(s)$ is implemented by the CPU, and the complementary sensitivity function, the transfer function from the reference position $u$ to $x$ in Fig. 4, is measured. Figure 8 shows the $-3 \mathrm{~dB}$ bandwidth of $3.5 \mathrm{kHz}$. Additionally, $x$ is measured when $u$ is set to the origin. Figure 9(a) shows that $x$ fluctuates within $\pm 2.5 \mathrm{~nm}$ only, resulting in a positioning resolution of $0.8 \mathrm{~nm}_{\mathrm{rms}}$, which is mainly given by the sensor resolution.

For further evaluation, step response of $x$ is measured by varying the step height, as shown in Fig. 9(b). Due to the achieved high positioning resolution, at least a step of $2.5 \mathrm{~nm}$ can be clearly resolved. In summary, the experiments validate that $C_{P}(s)$ rejects disturbances such as thermal drift, hysteresis and floor vibrations well, enabling high-precision positioning.

\section{Motion Trajectory}

Among different types of motion trajectories, a triangular wave is most commonly used for high-resolution imaging such as AFMs ${ }^{(26)}$. Thus, it is selected as the motion trajectory $r$ in Fig. 4, which can be described in the form of Fourier series ${ }^{(27)}$

$$
r(t)=\sum_{k=1}^{l}\left(r_{k} e^{j 2 \pi k f_{r} t}+r_{k}^{*} e^{-j 2 \pi k f_{r} t}\right), \cdots \cdots \cdots \cdots \cdots \cdots
$$


with the complex Fourier coefficient $r_{k}$ and its complex conjugate $r_{k}^{*}$

$$
r_{k}=-j \frac{4 A_{r}}{\pi^{2} k^{2}}\left\{\begin{array}{ll}
(-1)^{(k-1) / 2} & \text { for odd } k, \\
0 & \text { for even } k,
\end{array} .\right.
$$

where $A_{r}=1 \mu \mathrm{m}, f_{r}=400 \mathrm{~Hz}$ and $l=9$ are the amplitude, the frequency and the highest harmonics, respectively. These values are determined at the implementation such that the output voltage and current of the amplifier do not saturate.

The triangular wave has a slope between turning points. This part can be regarded as a ramp input, which Type 1 systems cannot track ${ }^{(25)}$. In other words, even if their triangular trajectory has an extremely low frequency, flexure-guided scanners with a PID or PI controller are incapable of perfect tracking and will follow this ramp signal with a constant delay. Thus, feedforward control is used for the scanner with the hybrid reluctance actuator to accurately track the triangular trajectory $r$ in the next section.

\section{Feedforward Control Design}

Feedforward control design typically requires accurate models for high-speed motion ${ }^{(28)}$. However, hybrid reluctance actuators exhibit hysteresis as demonstrated in Section 5.2, and obtaining its accurate model can be difficult and time-consuming, because it may depend on the temperature and the trajectory ${ }^{(29)}$. To take an advantage of the periodic scanning motion, however, modeling-free inversion-based iterative control (IIC) ${ }^{(27)}$ is used as feedforward control for the scanner with the hybrid reluctance actuator.

Similar to iterative learning control (ILC) ${ }^{(30)}$, modelingfree IIC improves the control input by learning from the tracking error in the previous trial such that the error is decreased in the next trial. Unlike ILC, however, learning can take place only at selected fundamental and harmonic frequencies of the trajectory to filter the sensor noise between the harmonics ${ }^{(27)}$ and to decrease the computation efforts ${ }^{(31)}$. Another advantage of modeling-free IIC is that it simultaneously updates its internal plant model. Thus, a model is not required for the operation, unlike typical feedforward control.

Modeling-free IIC is implemented as shown in Fig. 4. To activate learning only at the $r$ 's fundamental and harmonic frequencies, the mover position $x$, its input $u$ and $r$ are described by vectors

$$
\begin{aligned}
& U=\left[u_{1} \ldots u_{k} \ldots u_{l}\right]^{T}, X=\left[x_{1} \ldots x_{k} \ldots x_{l}\right]^{T} \\
& R=\left[r_{1} \ldots r_{k} \ldots r_{l}\right]^{T}, \ldots \ldots \ldots \ldots \ldots \ldots \ldots \ldots \ldots \ldots \ldots
\end{aligned}
$$

containing $k$-th complex Fourier coefficients $x_{k}, u_{k}$ and $r_{k}$ of $x(t), u(t)$ and $r(t)$, respectively. Note that the vector size $l$ determines the bandwidth of the modeling-free IIC, which is given by $l f_{r}=3.6 \mathrm{kHz}$.

The learning law at the $i$-th trial is given by ${ }^{(27)}$

$$
U_{i+1}=U_{i}+J_{i}^{-1}\left(R-X_{i}\right)
$$

where the matrix $J_{i}$ is the internal plant model. For fast learning and high tracking performance, it is updated by the Secant method $^{(27)(32)(33)}$, where $J_{i}$ is given by a diagonal matrix, and its $k$-th diagonal element $j_{k, i}$ is corrected by

$$
j_{k, i}= \begin{cases}\frac{x_{k, i}-x_{k, i-1}}{u_{k, i}-u_{k, i-1}} & \text { for }\left|u_{k, i}-u_{k, i-1}\right| \geq \epsilon \\ j_{k, i-1} & \text { otherwise. }\end{cases}
$$

The threshold $\epsilon$ is set to $1 \mathrm{~nm}$ to terminate the model update for stable learning ${ }^{(27)}$. The updated input $U_{i+1}$ is transformed to a time-domain signal and fed to the closed-loop system as $u$ for the next trial.

\section{Experimental Results}

To demonstrate the $400 \mathrm{~Hz}$ triangular scanning motion of $2 \mu \mathrm{m}$, the actuator position $x$ and the tracking error $r-x$ are measured with modeling-free IIC. For comparison, they are also measured without modeling-free IIC by using $r$ as $u$, as shown in Fig. 10. Without modeling-free IIC in Fig. 10(a), the turning motion excites oscillations that slowly decay. This is because $r$ has the frequency components between $400 \mathrm{~Hz}$ and $3.6 \mathrm{kHz}$, and the complementary sensitivity function in Fig. 8 has a magnitude higher than $0 \mathrm{~dB}$ beyond $400 \mathrm{~Hz}$. Consequently, Fig. 10(b) shows a tracking error of about $\pm 260 \mathrm{~nm}$ with an RMS value of $101 \mathrm{~nm}$.

In Fig. 10(c), modeling-free IIC significantly decreases the large error by a factor of 26 to $3.8 \mathrm{~nm}_{\mathrm{rms}}$, successfully demonstrating its effectiveness. For analysis of the residual tracking error, Fig. 10(d) shows its spectrum calculated by using Fast Fourier Transform. It can be seen that the error

(a)
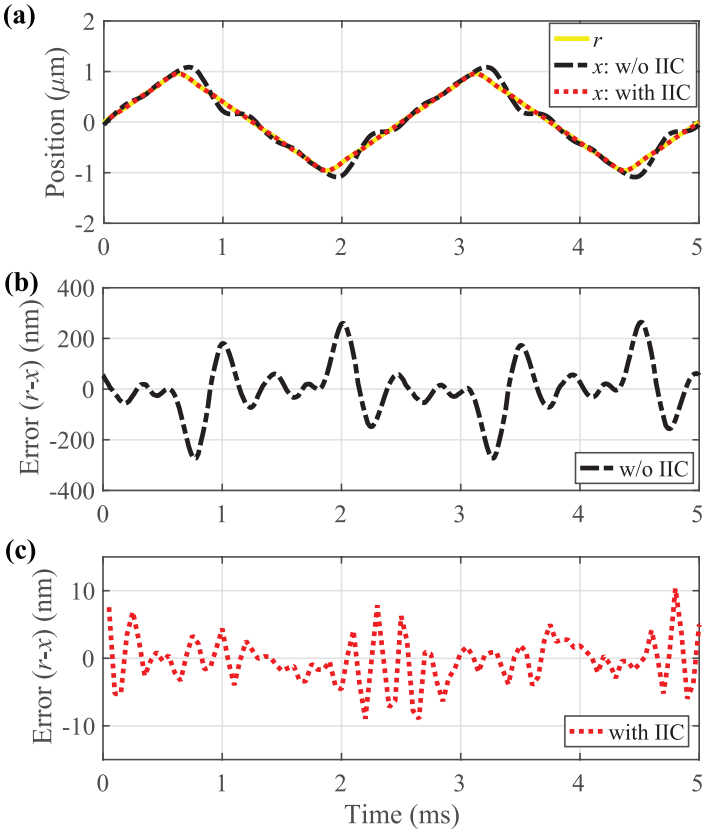

(d)

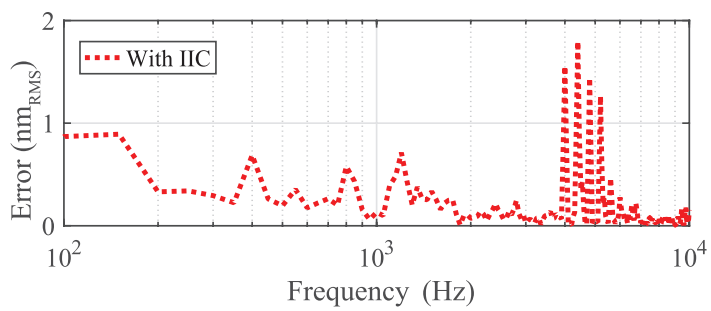

Fig. 10. $400 \mathrm{~Hz}$ scanning motion of $2 \mu \mathrm{m}$ : (a) measured position $x$ and motion trajectory $r$, (b)(c) tracking error $(r-x)$ when the scanner is operated by the position loop with and without modeling-free IIC, and (d) spectrum of the error with modeling-free IIC 
is well-compensated below $1 \mathrm{~nm}_{\mathrm{RMS}}$ at the frequencies approximately below $4 \mathrm{kHz}$. However, it is larger at higher frequencies. This would be because the hysteresis deforms the mover's trajectory and creates harmonics higher than $l=9$, as seen with other types of precision actuators (e.g. piezoelectric actuators $)^{(32)}$. Due to its bandwidth of $3.6 \mathrm{kHz}$, the modelingfree IIC does not compensate for the higher harmonics. This problem will be solved as a part of future work. Future work also includes further investigation of hybrid reluctance actuators for large motion as very first results can be found in ${ }^{(19)}$.

Overall, Fig. 10 clearly demonstrates that the proposed scanner based on the hybrid reluctance force is capable of high-speed scanning with nanometer resolution by using cascade control and modeling-free IIC.

\section{Conclusion}

For high-speed scanning motion with nanometer resolution, a scanner using a hybrid reluctance actuator is proposed. Cascade control consisting of current and position loops is designed, achieving a control bandwidth of $3.5 \mathrm{kHz}$ and a positioning resolution of $0.8 \mathrm{~nm}$. To track the $400 \mathrm{~Hz}$ triangular signal of $2 \mu \mathrm{m}$, modeling-free IIC is selected as feedforward control. The experimental results confirm the effectiveness of modeling-free IIC by significantly decreasing the tracking error from $101 \mathrm{~nm}$ to $3.8 \mathrm{~nm}$. As a result, this paper successfully demonstrates that the hybrid reluctance force is capable of high-speed scanning motion with nanometer resolution.

\section{Acknowledgment}

The financial support by the Christian Doppler Research Association, the Austrian Federal Ministry for Digital and Economic Affairs, the National Foundation for Research, Technology and Development, MICRO-EPSILON MESSTECHNIK GmbH \& Co. KG and ATENSOR Engineering and Technology Systems GmbH is gratefully acknowledged.

\section{References}

( 1 ) B. Zhang, J. He, X. Li, F. Xu, and D. Li: "Micro/nanoscale electrohydrodynamic printing: from 2D to 3D", Nanoscale, Vol.8, pp.15376-15388 (2016)

( 2 ) G. Schitter, K.J. Astrom, B.E. DeMartini, P.J. Thurner, K.L. Turner, and P.K. Hansma: "Design and modeling of a high-speed afm-scanner", IEEE Transactions on Control Systems Technology, Vol.15, pp.906-915 (2007)

( 3 ) S. Ito, S. Unger, and G. Schitter: "Vibration isolator carrying atomic force microscopes head", Mechatronics, Vol.44, pp.32-41 (2017)

( 4 ) G. Cherubini, C.C. Chung, W.C. Messner, and S.O.R. Moheimani: "Control methods in data-storage systems", IEEE Transactions on Control Systems Technology, Vol.20, pp.296-322 (2012)

( 5 ) S. Ito and G. Schitter: "Comparison and classification of high-precision actuators based on stiffness influencing vibration isolation", IEEE/ASME Transactions on Mechatronics, Vol.21, pp.1169-1178 (2016)

( 6 ) S. Ito, J. Steininger, and G. Schitter: "Low-stiffness dual stage actuator for long rage positioning with nanometer resolution", Mechatronics, Vol.29, pp.46-56 (2015)

( 7 ) M. Heertjes and G. Leenknegt: "Switching control in blu-ray disk drives", Mechatronics, Vol.20, No.4, pp.453-463 (2010)

( 8 ) S. Ito and G. Schitter: "Atomic force microscopy capable of vibration isolation with low-stiffness z-axis actuation”, Ultramicroscopy, Vol.186, pp.9-17 (2018)

( 9 ) G. Parmar, K. Barton, and S. Awtar: "Large dynamic range nanopositioning using iterative learning control”, Precision Engineering, Vol.38, No.1, pp.48-56 (2014)

(10) R. Munnig Schmidt, G. Schitter, A. Rankers, and J. van Eijk: The Design of High Performance Mechatronics, Delft University Press, 2nd revised ed. (2014)
(11) D.B. Hiemstra, G. Parmar, and S. Awtar: "Performance tradeoffs posed by moving magnet actuators in flexure-based nanopositioning", IEEE/ASME Transactions on Mechatronics, Vol.19, pp.201-212 (2014)

(12) T. Tuma, W. Haeberle, H. Rothuizen, J. Lygeros, A. Pantazi, and A. Sebastian: "Dual-stage nanopositioning for high-speed scanning probe microscopy", IEEE/ASME Transactions on Mechatronics, Vol.19, pp.10351045 (2014)

(13) A. Katalenic, H. Butler, and P.P.J. van den Bosch: "High-precision force control of short-stroke reluctance actuators with an air gap observer", IEEE/ASME Transactions on Mechatronics, Vol.21, pp.2431-2439 (2016)

(14) X. Lu: Electromagnetically-driven ultra-fast tool servos for diamond turning, $\mathrm{PhD}$ thesis, Massachusetts Institute of Technology (2005)

(15) D. Wu, X. Xie, and S. Zhou: "Design of a normal stress electromagnetic fast linear actuator", IEEE Transactions on Magnetics, Vol.46, pp.1007-1014 (2010)

(16) D. Laro, J. Dams, and J. van Eijk: "Linear hybrid actuator for active force cancellation", in International Symposium on Linear Drives for Industry Applications, p.244 (2011)

(17) D.J. Kluk, M.T. Boulet, and D.L. Trumper: "A high-bandwidth, highprecision, two-axis steering mirror with moving iron actuator", Mechatronics, Vol.22, No.3, pp.257-270 (2012) Special Issue on Mechatronic Systems for Micro- and Nanoscale Applications.

(18) E. Csencsics, J. Schlarp, and G. Schitter: "Bandwidth extension of hybridreluctance-force-based tip/tilt system by reduction of eddy currents", in IEEE International Conference on Advanced Intelligent Mechatronics, pp.11671172 (2017)

(19) S. Ito, S. Troppmair, B. Lindner, F. Cigarini, and G. Schitter: "Long-range fast nanopositioner using nonlinearities of hybrid reluctance actuator for energy efficiency (In press)", IEEE Transactions on Industrial Electronics (2018)

(20) S. Ito, F. Cigarini, S. Unger, and G. Schitter: "Flexure design for precision positioning using low-stiffness actuators", IFAC-PapersOnLine, Vol.49, No.21, pp.200-205 (2016) IFAC Symposium on Mechatronic Systems.

(21) A. Matsubara: Design and Control of Precision Positioning and Feed Drive Systems (in Japanese), Morikita Publishing Co., Ltd. (2008)

(22) T. Yamaguchi, M. Hirata, and C. Pang: High-Speed Precision Motion Control, Taylor \& Francis (2011)

(23) E.P. Furlani: Permanent Magnet and Electromechanical Devices, Academic Press (2001)

(24) K.A. Yi and R.J. Veillette: "A charge controller for linear operation of a piezoelectric stack actuator", IEEE Transactions on Control Systems Technology, Vol.13, pp.517-526 (2005)

(25) J. Van de Vegte: Feedback Control Systems, Prentice Hall, 3rd ed. (1994)

(26) T. Tuma, A. Sebastian, J. Lygeros, and A. Pantazi: "The four pillars of nanopositioning for scanning probe microscopy: The position sensor, the scanning device, the feedback controller, and the reference trajectory", IEEE Control Systems, Vol.33, pp.68-85 (2013)

(27) S. Ito, H.W. Yoo, and G. Schitter: "Comparison of modeling-free learning control algorithms for galvanometer scanner's periodic motion", in IEEE International Conference on Advanced Intelligent Mechatronics, pp.13571362 (2017)

(28) K.K. Leang, Q. Zou, and S. Devasia: "Feedforward control of piezoactuators in atomic force microscope systems", IEEE Control Systems Magazine, Vol.29, pp.70-82 (2009)

(29) K.M. Chowdary and S.A. Majetich: "Frequency-dependent magnetic permeability of Fe10Co90 nanocomposites", Journal of Physics D: Applied Physics, Vol.47, No.17, p.175001 (2014)

(30) D.A. Bristow, M. Tharayil, and A.G. Alleyne: "A survey of iterative learning control”, IEEE Control Systems Magazine, Vol.26, pp.96-114 (2006)

(31) M. Hehn and R. D'Andrea: “A frequency domain iterative learning algorithm for high-performance, periodic quadrocopter maneuvers", Mechatronics, Vol.24, No.8, pp.954-965 (2014)

(32) Y. Li and J. Bechhoefer: "Model-free iterative control of repetitive dynamics for high-speed scanning in atomic force microscopy", Review of Scientific Instruments, Vol.80, No.1, p.013702 (2009)

(33) Z. Wang and Q. Zou: "A modeling-free differential-inversion-based iterative control approach to simultaneous hysteresis-dynamics compensation", in American Control Conference, pp.3558-3563 (2015) 
Shingo Ito (Non-member) received the MASc in Mechanical and In-

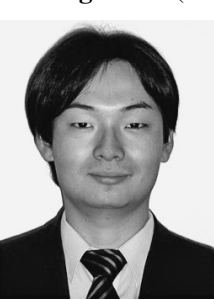
dustrial Engineering from the University of Toronto, Canada, in 2007 and the Ph.D. in Electrical Engineering from TU Wien, Vienna, Austria in 2015. From 2007 to 2010, he served as an engineer in the field of motion control at Yaskawa Electric Corporation, Iruma, Saitama, Japan. He is currently a postdoctoral researcher at the Automation and Control Institute (ACIN), TU Wien. His research interest includes design and control of high-precision mechatronic sys-

tems for production, inspection and automation.

Stefan Troppmair (Non-member) received the M.Sc. in Automation Technology in 2017 from TU Wien, Vienna Austria. His research for the Master's degree focused on the design and control of high-precision actuators at the Automation and Control Institute (ACIN), TU Wien Currently he serves as a software development engineer at Bosch Engineering $\mathrm{GmbH}$, Vienna Austria, developing motor control units for combustion and hybrid cars.
Francesco Cigarini (Non-member) is a Ph.D. student at the $\mathrm{Au}$ -

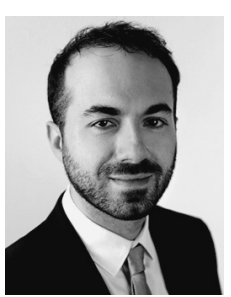
tomation and Control Institute (ACIN) of TU Wien, Vienna, Austria. He received the M.Sc. degree in Mechatronic Engineering from the University of Modena and Reggio Emilia, Reggio Emilia, Italy (2012). His research interest includes the design of mechatronic systems, inline metrology systems and multiphysics FEM analysis of electro-mechanical systems.

Georg Schitter (Non-member) is Professor at the Automation and

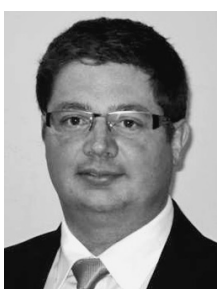
Control Institute (ACIN) of TU Wien. He received the M.Sc. in Electrical Engineering from TU Graz, Austria (2000) and the $\mathrm{PhD}$ degree from ETH Zurich, Switzerland (2004). His primary research interests are on high-performance mechatronic systems and multidisciplinary systems integration, particularly for precision engineering applications in the high-tech industry, scientific instrumentation, and mechatronic imaging systems, such as scanning probe microscopy, adaptive optics, and lithography systems for semiconductor industry. $\mathrm{He}$ serves as Associate Editor for the IEEE/ASME Transactions on Mechatronics (2010-2014) as well as for the IFAC Journals Control Engineering Practice and IFAC Mechatronics. 